This item was submitted to Loughborough's Research Repository by the author.

Items in Figshare are protected by copyright, with all rights reserved, unless otherwise indicated.

\title{
Development of an expert based ICD-9-CM and ICD-10-CM map to AIS 2005 update 2008
}

\section{PLEASE CITE THE PUBLISHED VERSION}

https://doi.org/10.1080/15389588.2016.1191069

\section{PUBLISHER}

Taylor \& Francis

\section{VERSION}

AM (Accepted Manuscript)

\section{PUBLISHER STATEMENT}

This is an Accepted Manuscript of an article published by Taylor \& Francis in Traffic Injury Prevention on 2 September 2016, available online: http://www.tandfonline.com/10.1080/15389588.2016.1191069.

\section{LICENCE}

CC BY-NC-ND 4.0

\section{REPOSITORY RECORD}

Loftis, Kathryn L., Janet P. Price, Patrick J. Gillich, Kathy J. Cookman, Amy L. Brammer, Trish St. Germain, Jo Barnes, et al.. 2016. "Development of an Expert Based ICD-9-CM and ICD-10-CM Map to AIS 2005 Update 2008”. Loughborough University. https://hdl.handle.net/2134/21577. 


\title{
Development of an Expert Based ICD-9-CM and ICD-10-CM Map to AIS 2005 Update 2008
}

Kathryn L. Loftis ${ }^{1}$, Janet P. Price ${ }^{1}$, Patrick J. Gillich ${ }^{1}$, Kathy J. Cookman ${ }^{1,2}$, Amy L. Brammer ${ }^{1}$, Trish St. Germain ${ }^{1}$, Lauren Jones $^{1,3}$, Jo Barnes ${ }^{1,4}$, Vickie Graymire ${ }^{1}$, Donna A. Nayduch ${ }^{1,5}$, Christine Read-Allsopp ${ }^{1,6}$, Kathy Baus ${ }^{1}$, Patsye A. Stanley ${ }^{1}$, Maureen Brennan ${ }^{1}$

\author{
${ }^{1}$ AAAM \\ 35 E. Wacker Dr Suite 850 \\ Chicago, IL 60601 \\ Lead Author Correspondence: klloftis@gmail.com, 410-306-0344 \\ ${ }^{2}$ KJ Trauma Consulting LLC \\ PO Box 4737 Fort Myers, FL 33918 \\ Kathy.Cookman@KJConsulting.us \\ ${ }^{3}$ Lauren Jones Consulting \\ Sydney, Australia \\ laurenj@ozemail.com.au \\ ${ }^{4}$ Design School \\ Loughborough University \\ Ashby Road, Loughborough LE11 3TU \\ ${ }^{5}$ AVP Trauma, HCA North FL Division \\ 315 NW 113th Cir. \\ Ocala, FL 34482 \\ ${ }^{6}$ Royal North Shore Hospital \\ Sydney, Australia \\ christine.allsopp@health.nsw.gov.au
}

\section{ABSTRACT}

Objective: This paper describes how maps were developed from the Clinical Modifications of the 9th and 10th Revisions of the International Classification of Diseases (ICD) to the Abbreviated Injury Scale 2005 Update 2008 (AIS08). The development of the mapping methodology is described, with discussion of the major assumptions used in the process to map ICD codes to AIS severities. There were many intricacies to developing the maps, since the 
two coding systems, ICD and AIS, were developed for different purposes and contain unique classification structures to meet these purposes.

Methods: Experts in ICD and AIS analyzed the rules and coding guidelines of both injury coding schemes to develop rules for mapping ICD injury codes to the AIS08. This involved subject matter expertise, detailed knowledge of anatomy, and an in-depth understanding of injury terms and definitions as applied in both taxonomies. The official ICD-9-CM and ICD-10-CM versions (injury sections) were mapped to the AIS08 codes and severities, following the rules outlined in each coding manual. The panel of experts was comprised of coders certified in ICD and/or AIS from around the world. In the process of developing the map from ICD to AIS, the experts created rules to address issues with the differences in coding guidelines between the two schemas and assure a consistent approach to all codes.

Results: Over 19,000 ICD codes were analyzed and maps were generated for each code to AIS08 chapters, AIS08 severities, and ISS body regions. After completion of the maps, 14,101 (74\%) of the eligible 19,012 injury related ICD-9-CM and ICD-10-CM codes were assigned valid AIS08 severity scores between 1 and 6. The remaining 4,911 codes were assigned an AIS08 of 9 (unknown) or were determined to be non-mappable because the ICD description lacked sufficient qualifying information for determining severity according to AIS rules. There were also 15,214 (80\%) ICD codes mapped to AIS08 chapter and ISS body region, which allow for ISS calculations for patient datasets.

Conclusion: This mapping between ICD and AIS provides a comprehensive, expert-designed solution for analysts to bridge the data gap between the injury descriptions provided in hospital codes (ICD-9-CM, ICD-10-CM) and injury severity codes (AIS08). By applying consistent rules from both the ICD and AIS taxonomies, the expert panel created these definitive maps, which are the only ones endorsed by AAAM. Initial validation upheld the quality of these maps for the estimation of AIS severity, but future work should include verification of these maps for MAIS and ISS estimations with large datasets. These ICD-AIS maps will support data analysis from databases with injury information classified in these two different systems and open new doors for the investigation of injury from traumatic events using large injury datasets.

\section{INTRODUCTION}

Hospitals across the world use International Classification of Diseases (ICD) codes, developed by the World Health Organization, to record diseases, injury diagnoses, and procedures for patients. Injury researchers and trauma centers typically use the Abbreviated Injury Scale (AIS) to code traumatic injuries and identify injury severity (AAAM 2008). AIS severities are then used to calculate the Injury Severity Score (ISS), which is used by trauma hospitals in many countries, including the United States (US), to compare severities and patient outcomes. There is a growing need for a map to bridge the gap between hospital databases using ICD and injury research databases using AIS, which would allow the large amounts of available ICD data to be used in a consistent manner for injury research. This paper presents the development of a map from ICD codes to AIS chapters, AIS severities, and ISS body regions for use in mapping large ICD datasets into severity metrics for use in trauma research. 
Other research groups have attempted to map from various database injury codes to AIS. One such group from Switzerland developed a map between police-perceived injury severities and AIS severities, but found it was a difficult process. They found that police severities were unreliable and many rules had to be in place in order to perform a standardized mapping (Schmitt et al. 2014).

Barnard et al created an AIS 2005 Update 2008 (AIS08) map to ICD-9-Clinical Modifications (CM) codes as mapped by coders within the Crash Injury Research and Engineering Network (CIREN) database. Given that ICD and AIS were created with differing levels of specificity based on their purposes, this work found that sometimes one ICD code mapped to multiple AIS codes and vice versa. For their map, the most frequent pairs were chosen using the CIREN database, but this meant the map was relevant specifically for motor vehicle crashes. Additionally, the coding used for creating this map was performed by coders who were not certified in either ICD or AIS (Barnard et al. 2012).

In the US, AIS training is not part of current medical coder training and requires a specially trained individual for manual coding, so there is a need to map from ICD to obtain ISS severities for hospital tracking. Since the calculation of ISS requires knowledge of AIS severities and the correct assignment of the AIS code to ISS body regions, a map from ICD would ideally need to provide information about both AIS chapters and severities that link to ISS body regions. Bartolomeo et al reviewed an Italian-derived program known as ICD Programs for Injury Characterization (ICDPIC) that mapped from ICD-9-CM to AIS 1990 and compared that mapping to expert-coded cases in AIS, also calculating the ISS. The researchers found that ICDPIC performed poorly at properly mapping ISS values, and often under-coded a patient's true injury severity. One problem was that no more than six ICD-9CM codes were recorded per patient, which potentially limited the number of injuries that were properly recorded to include in the mapping. Consequently, there was only a $12 \%$ agreement found between the ICDPIC and expert coded severities (Bartolomeo et al. 2010). Bellengham et al compared severity metrics from ICD-9-CM to AIS severity mapped using ICDPIC. They found that the metrics based on ICD-9-CM, which was originally reported, out-performed metrics derived from the mapped AIS severities (Bellengham et al. 2016). Given the Bartolomeo study results where ICDPIC performed poorly in mapping to AIS, a revised Bellingham study may reveal better AIS results with an updated mapping program. Garthe et al detailed a list of specifications that would need to be considered if creating a map between ICD and AIS. They noted that a map was needed, since large ICD datasets need to be converted into AIS but this would be time-consuming and expensive to complete manually (Garthe et al. 1999). A programmatic mapping between the coding systems would provide a quick and reproducible method for converting entire databases. Similar findings were reported in a World Health Organization bulletin, which advocated for the creation of ICD codes with severity metrics (Nakahara et al. 2011). Linking between ICD and the injury severities associated with AIS would be helpful for assessing injury treatment and survivability across the globe.

One of the most well-known historical maps between ICD and AIS is the ICDMAP90 program. This map was previously used by the US National Trauma Data Bank (NTDB) for conversion of injury codes within their trauma database. The ICDMAP90 program requires the user to select either "high" or "low" severity options, which makes repeatability of mapping more difficult when it is unknown which choice was used for mapping. It also results in 
many generic (less severe) AIS 1990 codes when mapping from ICD-9-CM. Finally, the ICDMAP90 program is now seriously outdated given the new revisions of ICD-10-CM and AIS08.

As the previous studies concluded, mapping from ICD to AIS requires experts in both coding systems, development of rules for consistent, systematic mapping, and creation of a map that is available for use globally. The objective of this work was the development of such a map between ICD (versions 9 and 10) and AIS 2005 Update 2008.

\section{METHODS}

The official ICD-9-CM and ICD-10-CM versions were used for mapping ICD codes to AIS08 codes and severities (Hart 2014, Schmidt 2015, AAAM 2008). ICD-10-CM has much greater specificity compared to ICD-9-CM, as evidenced by the increased number of codes in the later version. Only the injury sections of each ICD version were considered for this project (800-999 in ICD-9-CM, S and T sections in ICD-10-CM). ICD injury codes are organized according to type of injury (fracture, internal organ, etc) and general body region injured. As an example of the ICD organization, ICD-9-CM contains 182 skull fracture codes with and without brain injury from codes 800 to 801.99 . ICD-10-CM greatly changed the organization of codes, where S02-S02.92 includes 108 skull and facial bone fracture codes, without inclusion of brain injury. In comparison, AIS08 has 13 possible codes devoted to skull fractures (not including the crush code) within the Head Chapter and brain injuries are coded separately. As this example shows, correct mapping required a great deal of knowledge in ICD-9-CM, ICD-10-CM, and AIS08. To perform the mapping, an international panel of coders certified in either ICD or AIS (or both) was recruited. These experts analyzed the coding rules and guidelines of both injury coding schemes to develop rules for mapping ICD injury codes to the AIS08). This involved subject matter expertise, detailed knowledge of anatomy, and an in-depth understanding of injury terms and definitions as applied in both of the taxonomies.

The maps were developed in a multi-step process, following the rules outlined in each coding manual. The mapping for ICD-9-CM was completed first and the rules and procedures from that map were used to develop the ICD-10CM map. Initially, each ICD code was reviewed and the most appropriate AIS08 codes for each were selected. This required expert knowledge of both coding systems. As an example, ICD codes specifying "crush" injuries do not meet the requirements for coding "crush" within AIS08, therefore, ICD crush codes could not be mapped to AIS08 crush codes and typically resulted in either a generic map or no map. For ICD codes described as "other specified”, previously unused valid specific AIS08 codes were used for the map, whereas for ICD codes described as "other unspecified" the appropriate "not further specified" AIS08 code was chosen for the map. When there was a choice between two possible AIS08 codes of different severities, the AIS rule of "code conservatively" was followed and the lower AIS severity code was chosen. Depending on the ICD code, sometimes multiple AIS08 codes were required to fulfill the map, so the Boolean operator "AND” was used. For some ICD codes, different AIS08 codes could fulfill the map, and for these the Boolean operator "OR" was used. An example is the ICD-9-CM code 800.11 "Closed fracture of vault of skull with cerebral laceration and contusion; with no loss of consciousness". In ICD, the "and" in the description is to be interpreted as an "or", so when mapping to AIS08, a user would need to code the closed vault skull fracture (150402.2) AND the cerebral laceration (140688.3) OR contusion (140602.3). Head injuries within the ICD posed 
unique challenges for mapping. Where an ICD code specified skull fracture, brain injury, and loss of consciousness (LOC), AIS08 rules for coding LOC were followed. When there was a documented anatomic brain injury, the loss of consciousness was not coded in addition to the brain injury.

After all ICD codes were paired with AIS08 codes, the maps were reviewed. Each code map was studied to ensure it followed the mapping rules. A one-to-one mapping between ICD and AIS08 codes was not possible given the significant differences in specificity and coding structure within the two systems. Recognizing the requirement for the mapping to result in the ability to generate an ISS, a map was created that provided the AIS severity, AIS chapter, and ISS body region for each ICD code. There are 6 possible post-dot severities within AIS08, ranging from 1, which is minor (such as external contusion), to 6 which is maximal (such as decapitation). An AIS post-dot score of 9 indicates that no severity could be assigned. While there are 9 AIS08 Chapters (listed in Table 1), with each addressing a separate part of the body, there are only 6 body regions defined by ISS (Head and Neck, Face, Chest, Abdominal or pelvic contents, Extremities or pelvic girdle, External). There are rules provided within the AIS dictionary indicating how each AIS code maps to an ISS body region. In this way, creating a map between an ICD code and an AIS code then allowed an ISS body region to be assigned following the rules already established by the AIS coding dictionary. Sometimes an ICD code mapped to AIS08 codes in the same body region with differing severities, in which case the decision was made to select the maximum AIS08 severity for ISS purposes. This decision was based on the fact that the severities would be used to calculate ISS and under-coding would not appropriately convey the potential injury severity for the patient. In selecting the multiple AIS08 codes that originally mapped to the one ICD code, the AIS08 "code conservatively" rule was followed, so each of the AIS08 codes that mapped were already the lowest possible severity for that map. Referring back to the previous example where one ICD code mapped to multiple AIS codes, closed vault skull fracture (150402.2) AND cerebral laceration (140688.3) OR contusion (140602.3), the AIS08 severity selected would be a " 3 " instead of "2", as selecting the 2 would not properly communicate the severity of the overall injury sustained by the patient. When ICD codes mapped to AIS08 codes from multiple chapters, a precedence order was established based on threat to life and vital organs. These chapters are listed in Table 1 in decreasing order of precedence.

Table 1. AIS08 Chapter Mapping Precedence for ICD Codes that Mapped to Multiple AIS Codes

\begin{tabular}{|c|}
\hline $\begin{array}{c}\text { AIS08 Chapter Mapping } \\
\text { Precedence (Decreasing) }\end{array}$ \\
\hline Head - Chapter 1 \\
\hline Thorax - Chapter 4 \\
\hline Spine - Chapter 6 \\
\hline Abdomen - Chapter 5 \\
\hline Neck - Chapter 3 \\
\hline Lower Extremity - Chapter 8 \\
\hline Upper Extremity - Chapter 7 \\
\hline Face - Chapter 2 \\
\hline External - Chapter 9 \\
\hline Other Trauma - Chapter 9 \\
\hline
\end{tabular}


In this way, each ICD code was assigned an AIS08 chapter, ISS body region, and AIS08 severity.

\section{RESULTS}

Upon completion of the maps, 2,504 ICD-9-CM codes and 16,508 ICD-10-CM codes had been individually evaluated for maps to AIS08 and ISS. Of these, 2,202 ICD-9-CM and 13,102 ICD-10-CM could be assigned to an AIS08 chapter and ISS body region, as shown in Tables 2 and 3. There were more head codes found in ICD-9-CM than in ICD-10-CM, but the changes in ICD-10-CM greatly expanded the mappable codes for upper and lower extremities. Table 2 also displays the percentage of AIS codes in each chapter within the AIS08 dictionary. There are 1,999codes in the AIS 2005 Update 2008 dictionary, with the largest percentage of those (20\%) found in the lower extremity chapter.

Table 2. Mapping Results for ICD to AIS chapters

\begin{tabular}{|l|r|r|r|r|}
\hline \multicolumn{1}{|c|}{ ICD to AIS Chapters } & \multicolumn{2}{c|}{$\begin{array}{c}\text { ICD-9-CM to AIS08 } \\
\text { Chapters }\end{array}$} & \multicolumn{2}{c|}{$\begin{array}{c}\text { ICD-10-CM to AIS08 } \\
\text { Chapters }\end{array}$} \\
\hline $\begin{array}{l}\text { AIS08 Chapters and } \\
\text { Percentage of Each } \\
\text { Within Dictionary }\end{array}$ & \multicolumn{1}{|c|}{ Count } & \multicolumn{1}{c|}{ Percentage } & \multicolumn{1}{c|}{ Count } & Percentage \\
\hline No Map & 302 & $12.06 \%$ & 3496 & $21.18 \%$ \\
\hline Other Trauma (1.10\%) & 1 & $0.04 \%$ & 20 & $0.12 \%$ \\
\hline Head (14.06\%) & 504 & $20.13 \%$ & 345 & $2.09 \%$ \\
\hline Face (8.75\%) & 136 & $5.43 \%$ & 408 & $2.47 \%$ \\
\hline Neck (5.55\%) & 24 & $0.96 \%$ & 168 & $1.02 \%$ \\
\hline Thorax (9.55\%) & 98 & $3.91 \%$ & 464 & $2.81 \%$ \\
\hline Abdomen (12.51\%) & 191 & $7.63 \%$ & 574 & $3.48 \%$ \\
\hline Spine (10.81\%) & 122 & $4.87 \%$ & 716 & $4.34 \%$ \\
\hline Upper Extremity (16.26\%) & 398 & $15.89 \%$ & 4724 & $28.62 \%$ \\
\hline Lower Extremity (20.11\%) & 342 & $13.66 \%$ & 4307 & $26.09 \%$ \\
\hline External (1.30\%) & 386 & $15.42 \%$ & 1286 & $7.79 \%$ \\
\hline Total & 2504 & & 16508 & \\
\hline
\end{tabular}

A large percentage of the ISS body regions that mapped from ICD were in the Extremities and Pelvic Girdle, accounting for $26 \%$ of codes from ICD-9-CM and 49\% of codes from ICD-10-CM (Table 3). There was a larger percentage of ICD-10-CM codes that could not be mapped to an ISS body region (21\%) compared to those that couldn’t be mapped in ICD-9-CM (12\%). 
Table 3. Mapping Results for ICD to ISS Body Regions

\begin{tabular}{|l|r|r|r|r|}
\hline \multirow{2}{*}{ ICD to ISS Body Region } & \multicolumn{3}{|c|}{$\begin{array}{c}\text { ICD-9-CM to ISS Body } \\
\text { Regions }\end{array}$} & \multicolumn{2}{c|}{$\begin{array}{c}\text { ICD-CM to ISS Body } \\
\text { Regions }\end{array}$} \\
\hline ISS Body Regions & \multicolumn{1}{|c|}{ Count } & \multicolumn{1}{c|}{ Percentage } & \multicolumn{1}{c|}{ Count } & \multicolumn{1}{c|}{ Percentage } \\
\hline No Map & 302 & $12.06 \%$ & 3496 & $21.18 \%$ \\
\hline Head and Neck & 603 & $24.08 \%$ & 809 & $4.90 \%$ \\
\hline Face & 136 & $5.43 \%$ & 435 & $2.64 \%$ \\
\hline Chest & 131 & $5.23 \%$ & 478 & $2.90 \%$ \\
\hline $\begin{array}{l}\text { Abdominal and Pelvic } \\
\text { Contents }\end{array}$ & 206 & $8.23 \%$ & 687 & $4.16 \%$ \\
\hline $\begin{array}{l}\text { Extremities and Pelvic } \\
\text { Girdle }\end{array}$ & 656 & $26.20 \%$ & 8063 & $48.84 \%$ \\
\hline External & 470 & $18.77 \%$ & 2540 & $15.39 \%$ \\
\hline
\end{tabular}

As shown in Table 4, after completion of the maps, 14,101 of the 19,012 injury related ICD-CM codes were assigned valid AIS08 scores between 1 and 6. The remaining 4,911 codes were assigned an AIS severity of 9 or were determined to be non-mappable because the ICD description lacked sufficient qualifying information for determining severity according to AIS08 rules.

Table 4. Mapping Results from ICD to AIS08 Severities

\begin{tabular}{|c|r|r|r|r|}
\hline $\begin{array}{c}\text { ICD to AIS } \\
\text { Severity }\end{array}$ & \multicolumn{2}{|c|}{$\begin{array}{c}\text { ICD-9-CM to AIS08 } \\
\text { Severity }\end{array}$} & \multicolumn{2}{|c|}{$\begin{array}{c}\text { ICD-10-CM to AIS08 } \\
\text { Severity }\end{array}$} \\
\hline $\begin{array}{c}\text { AIS08 Severity } \\
\text { and Percentage } \\
\text { of Dictionary }\end{array}$ & Count & Percentage & Count & Percentage \\
\hline 9 and non-maps & 426 & $17.01 \%$ & 4485 & $27.17 \%$ \\
\hline $\begin{array}{c}1-\text { minor } \\
(22.36 \%)\end{array}$ & 557 & $22.24 \%$ & 4466 & $27.05 \%$ \\
\hline $\begin{array}{c}2 \text { - moderate } \\
\text { (36.47\%) }\end{array}$ & 738 & $29.47 \%$ & 5234 & $31.71 \%$ \\
\hline $\begin{array}{c}3 \text { - serious } \\
(20.96 \%)\end{array}$ & 415 & $16.57 \%$ & 1897 & $11.49 \%$ \\
\hline $\begin{array}{c}4-\text { severe } \\
(8.60 \%)\end{array}$ & 139 & $5.55 \%$ & 148 & $0.90 \%$ \\
\hline $\begin{array}{c}5-\text { critical } \\
(7.75 \%)\end{array}$ & 193 & $7.71 \%$ & 241 & $1.46 \%$ \\
\hline $\begin{array}{c}6 \text { - maximal } \\
(1.65 \%)\end{array}$ & 36 & $1.44 \%$ & 37 & $0.22 \%$ \\
\hline
\end{tabular}

\section{DISCUSSION}

There were intense discussions about procedures and rules among the expert panel during the mapping process. Initially, ICD and AIS coding rules were examined in detail before decisions were made by the experts in moving forward with mapping codes. Once decided and agreed upon, these became mapping rules that were then strictly followed throughout the mapping process so that a standardized approach was assured. The knowledge, experience, and expertise brought by each person within the group enabled the development of the highest quality map from ICD-9-CM and ICD-10-CM to AIS08. Given the level of complexity in the different coding systems and rules, this 
paper presents the first map to be developed by experts in both ICD and AIS using a manual technique for mapping each ICD code. As shown in the literature, previous maps developed using computer systems, surveys, or untrained coders could not achieve the level of complexity required to develop a map with an equivalent level of specificity. The maps created by this group are now available through AAAM and should be utilized when performing analysis on large datasets where comparison between ICD and AIS08 is required.

Initial rules were developed for mapping ICD-9-CM to AIS08. These rules were then applied to mapping ICD-10CM to AIS08, where some additional rules had to be developed to specifically address new types of codes found within ICD-10-CM. Map results were validated by comparing to survey work completed by Zonfrillo et al.

(Zonfrillo et al. 2015). For the survey, trauma professionals were instructed to assign AIS severity of 1 or 2, 3+, or indeterminable to ICD-9-CM and ICD-10-CM codes. Results from the validation showed strong agreement between the expert-derived maps and survey results. In each instance where survey results didn't agree with the expertderived map the expert map was determined to be the most appropriate map when following the rules that had been developed. In this way, every ICD code was re-analyzed to ensure the mapping rules were valid and appropriate. Maps from ICD were limited by the available codes in AIS08 for traumatic injury. There were over 2,500 ICD-9CM codes and over 16,500 ICD-10-CM to map, but there were only 1,999 AIS codes within the dictionary. Therefore, when completing the maps, some AIS08 codes were used more than once while some were never used, due to the specificity differences between ICD and AIS. ICD-10-CM greatly expanded the number and specificity of ICD codes and changed the organization of the codes compared to ICD-9-CM (Coster et al. 2006). Within ICD10-CM, there were large groups of codes that could not be mapped to AIS, including superficial foreign bodies, blisters, poisoning, and complications. These sections of ICD-10-CM accounted for the larger percentage of nonmappable codes when converting to AIS and ISS compared to ICD-9-CM.

A much larger percentage of ICD codes mapped to the AIS External chapter, compared to the percentage of AIS codes that are found within the chapter. ICD has many more codes describing external injuries such as lacerations, puncture wounds, contusions, and abrasions as compared to AIS. The lack of specificity in external (skin) injuries, with the exception of burns, in AIS is a reflection of the belief that these injuries rarely affect overall severity in multiple injury cases. As introduced in the methods, ICD codes for the head greatly changed in organization between ICD-9-CM and ICD-10-CM. In ICD-9-CM, skull fracture codes could be combined with brain injury codes. In ICD-10-CM, skull and facial fractures were one section while brain injuries were another. Therefore, the change in mappable AIS08 for the head is largely due to the change in the ICD versions and not a drop in mappable codes for skull and brain injuries in AIS08. Another interesting result was the percentage shift in ICD codes that mapped to AIS Upper and Lower Extremity Chapters between ICD-9-CM and ICD-10-CM. In ICD-9-CM, 30\% of mapped codes fell into the AIS Upper and Lower Extremity Chapters compared to over 50\% that mapped to AIS Upper and Lower Extremities for ICD-10-CM. This provides another example of the large changes in specificity in ICD-10-CM versus ICD-9-CM.

Similar trends were seen when investigating the results of ICD to ISS body regions. ICD codes mapping to the ISS Extremities and Pelvic Girdle went up over 20\% between ICD-9-CM and ICD-10-CM, whereas ICD codes mapped to the Head and Neck fell by almost 20\%. The ICD codes mapped to ISS External body region were similar 
between the two ICD versions, with ICD-9-CM at 19\% and ICD-10-CM at 15\% ISS External. The other ISS body regions that didn't greatly change between ICD versions were Face, Chest, and Abdomen and Pelvic Contents, but each comprised less than $10 \%$ of the respective maps.

The ICD-9-CM to AIS08 severity maps more closely matched the severity percentages in the AIS08 dictionary compared with results from ICD-10-CM. There were higher percentages of ICD-10-CM codes that could not be mapped to an AIS08 severity (27\%) compared to ICD-9-CM (17\%) while the percentages of the AIS 4+ severities were lower for ICD-10-CM compared to ICD-9-CM. For the ICD-9-CM codes mapped to AIS08 severities, AIS 4, 5, and 6 comprised $14.7 \%$ of the mapped codes, while for ICD-10-CM they were only $2.58 \%$ of codes. More analysis into the changes between ICD-9-CM and ICD-10-CM may be useful for elucidating the differences in the AIS08 maps for each one.

In summary, an expert group of ICD and AIS coders developed mapping rules following the coding guidelines of both schemas to create maps from ICD-9-CM and ICD-10-CM to AIS08. Over 19,000 ICD codes were analyzed and maps were generated for each code to AIS08 severities, AIS08 chapters, and ISS body regions. This work presents the first ICD-9-CM and ICD-10-CM maps to AIS08 to be developed by a group of expert certified coders on a code-by-code basis. It was validated by an independently created survey and should now be applied to large datasets for verification in calculating patient MAIS and ISS metrics for analysis and research. The maps will allow large hospital datasets coded with ICD to be compared to research datasets coded in AIS08 in an accurate and consistent manner.

\section{ACKNOWLEDGEMENTS}

The authors would like to acknowledge the AAAM Board for the support and sponsorship in developing these maps.

\section{REFERENCES}

1. AAAM. Abbreviated injury Scale 2005 update 2008, Association for the Advancement of Automotive Medicine, Chicago, Ill, 2008.

2. Barnard RT, Loftis KL, Martin RS, Stitzel JD. Development of a robust mapping between AIS 2+ and ICD-9-CM injury codes, Accident Analysis and Prevention, 2013, 52:133-43.

3. Bartolomeo SD, Tillati S, Valent F, Zanier L, Barbone F. ISS mapped from ICD-9-CM by a novel freeware versus traditional coding: a comparative study, Scandinavian Journal of Trauma, Resuscitation and Emergency Medicine, 2010; 18:17.

4. Bellegham GV, Devos S, De Wit L, Ives H, Lauwaert D, Pien K, Putnam K. Predicting in-hospital mortality of traffic victims: A comparison between AIS-and ICD-9-CM-related injury severity scales when only ICD-9-CM is reported, Injury: Int. J. Care Injured 2016; 47:141-146.

5. Coster CD, Quan J, Finlayson A, Gao M, Halfon P, Humphries KH, Johansen H, Lix LM, Luthi JC, Ma J, Romano PS, Roos L, Sundararajan Vijaya, Tu JV, Webster G, Ghali WA. Identifying priorities in methodological research using ICD-9-CM and ICD-10 administrative data: report from an international consortium, BMC Health Services Research 2006, 6:77. 
6. Garthe E, States JD, Mango NK. Abbreviated injury scale unification: The case for a unified injury system for global use, J. Trauma, 1999 Aug; 47(2):309-23.

7. Hart AC, ICD-9-CM for Hospitals - International Classification of Diseases $9^{\text {th }}$ Revision Clinical Modification Sixth Edition, OPTUM, 2014.

8. Nakahara S, Yokota J, Revision of the International Classification of Diseases to include standardized descriptions of multiple injuries and injury severity, Bulletin of the World Health Organization, 2011; 89:238-240.

9. Schmidt A, Schmidt K, Willard P. ICD-10-CM for Hospitals - International Classification of Diseases $10^{\text {th }}$ Revision Clinical Modification, OPTUM, 2015.

10. Schmitt KU, Baumgartner L, Muser M, Furter K, Scholz S, Luber B, Thomas P, Simma A. Developing a scheme to report AIS-coded injury severity for Swiss traffic accident data, IRCOBI Conference 2014.

11. Zonfrillo MR, Weaver AA, Gillich PJ, Price JP, Stitzel JD. New Methodology for an Expert-Designed Map From International Classification of Diseases (ICD) to Abbreviated Injury Scale (AIS) 3+ Severity Injury, Traffic Injury Prevention 2015, 16:S2. 